\title{
Water vapor in Titan's stratosphere from Cassini CIRS far-infrared spectra
}

\author{
V. Cottini ${ }^{\mathrm{a}, *}$, C.A. Nixon ${ }^{\mathrm{a}, \mathrm{b}}$, D.E. Jennings ${ }^{\mathrm{a}}$, C.M. Anderson ${ }^{\mathrm{a}}$, N. Gorius ${ }^{\mathrm{a}, \mathrm{c}}$, G.L. Bjoraker ${ }^{\mathrm{a}}$, A. Coustenis ${ }^{\mathrm{d}}$, \\ N.A. Teanby ${ }^{\mathrm{e}}$, R.K. Achterberg ${ }^{\mathrm{a}, \mathrm{b}}$, B. Bézard ${ }^{\mathrm{e}}$, R. de Kok $^{\mathrm{f}}$, E. Lellouch ${ }^{\mathrm{d}}$, P.G.J. Irwin ${ }^{\mathrm{g}}$, F.M. Flasar ${ }^{\mathrm{a}}$, \\ G. Bampasidis $\mathrm{d}, \mathrm{h}$
}

a Planetary Systems Laboratory, NASA Goddard Space Flight Center, Greenbelt, MD 20771, USA

${ }^{\mathrm{b}}$ Department of Astronomy, University of Maryland at College Park, College Park, MD 20742, USA

${ }^{\mathrm{c}}$ Department of Physics, The Catholic University of America, Washington, DC 20064, USA

${ }^{\mathrm{d}}$ LESIA-Observatoire de Paris, CNRS, UPMC Univ. Paris 06, Univ. Paris-Diderot, France

e School of Earth Sciences, University of Bristol, Wills Memorial Building, Queen's Road, Bristol BS8 1RJ, UK

${ }^{\mathrm{f}}$ SRON, Sorbonnelaan 2, 3584 CA Utrecht, Netherlands

${ }^{\mathrm{g}}$ Atmospheric, Oceanic and Planetary Physics, University of Oxford, Parks Rd., Oxford OX1 3PU, UK

${ }^{\mathrm{h}}$ Faculty of Physics, National and Kapodistrian University of Athens, Athens, Greece

\section{A R T I C L E I N F O}

\section{Article history:}

Received 17 April 2012

Revised 7 June 2012

Accepted 9 June 2012

Available online 26 June 2012

\section{Keywords:}

Spectroscopy

Atmospheres, Composition

Satellites, Atmospheres

Titan

\begin{abstract}
A B S T R A C T
Here we report the measurement of water vapor in Titan's stratosphere using the Cassini Composite Infrared Spectrometer (CIRS, Flasar, F.M. et al. [2004]. Space Sci. Rev. 115, 169-297). CIRS senses water emissions in the far infrared spectral region near $50 \mu \mathrm{m}$, which we have modeled using two independent radiative transfer codes (NEMESIS (Irwin, P.G.J. et al. [2008]. J. Quant. Spectrosc. Radiat. Trans. 109, 11361150) and ART (Coustenis, A. et al. [2007]. Icarus 189, 35-62; Coustenis, A. et al. [2010]. Icarus 207, 461476). From the analysis of nadir spectra we have derived a mixing ratio of $0.14 \pm 0.05 \mathrm{ppb}$ at an altitude of $97 \mathrm{~km}$, which corresponds to an integrated (from 0 to $600 \mathrm{~km}$ ) surface normalized column abundance of $3.7 \pm 1.3 \times 10^{14}$ molecules $/ \mathrm{cm}^{2}$. In the latitude range $80^{\circ} \mathrm{S}$ to $30^{\circ} \mathrm{N}$ we see no evidence for latitudinal variations in these abundances within the error bars. Using limb observations, we obtained mixing ratios of $0.13 \pm 0.04 \mathrm{ppb}$ at an altitude of $115 \mathrm{~km}$ and $0.45 \pm 0.15 \mathrm{ppb}$ at an altitude of $230 \mathrm{~km}$, confirming that the water abundance has a positive vertical gradient as predicted by photochemical models (e.g. Lara, L.M., Lellouch, F., Lopez-Moreno, J.J., Rodrigo, R. [1996]. J. Geophys. Res. 101(23), 261; Wilson, E.H., Atreya, S.K. [2004]. J. Geophys. Res. 109, E6; Hörst, S.M., Vuitton, V., Yelle, R.V. [2008]. J. Geophys. Res., 113, E10). We have also fitted our data using scaling factors of $\sim 0.1-0.6$ to these photochemical model profiles, indicating that the models over-predict the water abundance in Titan's lower stratosphere.
\end{abstract}

() 2012 Elsevier Inc. All rights reserved.

\section{Introduction}

Water is present in its various forms in many regions of the Solar System, from the atmospheres of the inner planets and shadows of lunar craters, to the mantles of icy satellites and beyond to the Kuiper Belt Objects (KBOs) and Oort Cloud Comets. Liquid water is also an essential ingredient for life on Earth and a potential clue in the search for life or habitability conditions in the rocks of Mars, the internal ocean of Europa or Titan, and the volcanic vents of Enceladus. On Titan, Saturn's largest satellite that hosts a dense nitrogen-dominated atmosphere, water is a trace species in the atmosphere. However, water plays a significant role since it is one of the sources of oxygen for the observed active photochemis-

* Corresponding author. Address: NASA/GSFC, Code 693, Bldg. 34, Rm. S121, 8800 Greenbelt Rd., Greenbelt, MD 20771, USA.

E-mail address: valeria.cottini@nasa.gov (V. Cottini). try on Titan (e.g. Lara et al., 1996; Wilson and Atreya, 2004; Hörst et al., 2008).

Titan's known oxygen compounds to date are carbon monoxide ( $\mathrm{CO}, \sim 47 \mathrm{ppm})$, carbon dioxide $\left(\mathrm{CO}_{2}, \sim 15 \mathrm{ppb}\right)$ and water vapor $\left(\mathrm{H}_{2} \mathrm{O}\right)$, where the abundances are quoted for the low-latitude stratosphere (de Kok et al., 2007a). $\mathrm{CO}_{2}$ was first detected by Voyager 1 (Samuelson et al., 1983), while CO was first seen by groundbased observations in the near-IR (Lutz et al., 1983). Subsequent observations in the sub-millimeter led to controversy as to whether CO was well-mixed or not (Hidayat et al., 1998; Gurwell, 2004). CO emission lines were later observed by the Cassini Composite Infrared Spectrometer (CIRS), thus improving the previous abundance estimate (de Kok et al., 2007a; Teanby et al., 2009). Water was first detected in Titan's atmosphere by the Infrared Space Observatory (ISO) in 1997 (Coustenis et al., 1998). Two lines near $40-\mu \mathrm{m}$ observed by the Short Wavelength Spectrometer (SWS) were modeled using a uniform mixing ratio above the condensation level and a value of $0.4 \mathrm{ppb}$ was detected. An early 
attempt to measure $\mathrm{H}_{2} \mathrm{O}$ with Cassini CIRS was unsuccessful due to poor signal-to-noise $(\mathrm{S} / \mathrm{N})$ ratios in early versions of the calibration pipeline spectra and a limited number of available spectra. Therefore, only an upper limit of $0.9 \mathrm{ppb}$ could be retrieved (de Kok et al., 2007a). Since then, water emission in the CIRS data has been definitely observed, albeit without deriving any further information on its abundance and distribution (Bjoraker et al., 2008). The Cassini Ion and Neutral Mass Spectrometer (INMS) detected $\mathrm{H}_{2} \mathrm{O}$ in the upper atmosphere - between 950 and $1200 \mathrm{~km}$ - with a mixing ratio in the range of $\sim(0.4-3.4) \times 10^{-5}$ (Cui et al., 2009).

While the presence of these oxygen compounds is now wellestablished, some details about their origin remain to be determined. Early photochemical models assumed that $\mathrm{CO}$ originated from episodic outgassing from Titan's interior along with nitrogen $\left(\mathrm{N}_{2}\right)$ or ammonia $\left(\mathrm{NH}_{3}\right)$ and methane $\left(\mathrm{CH}_{4}\right)$, whereas water molecules entered the top of the atmosphere and were photochemically dissociated to produce hydroxyl radicals $(\mathrm{OH})$ (Wong et al., 2002; Wilson and Atreya, 2004). The combination of $\mathrm{OH}$ and $\mathrm{CO}$ led to the production of $\mathrm{CO}_{2}$. However, Hörst et al. (2008) have recently challenged this model, arguing instead that both $\mathrm{CO}$ and $\mathrm{CO}_{2}$ are the result of upper-atmospheric chemistry that occurs between in-falling oxygen species reacting with carbon produced by $\mathrm{CH}_{4}$ photodissociation. In this hypothesis, water enters Titan's atmosphere either in the form of $\mathrm{H}_{2} \mathrm{O}$ or $\mathrm{OH}$ (since the latter is quickly converted to $\mathrm{H}_{2} \mathrm{O}$ within the atmosphere) together with oxygen ( $\mathrm{O}$ and $\mathrm{O}^{+}$). These forms of oxygen are thought to be deposited on Ti$\tan$ at two different altitudes. $\mathrm{O}^{+}$ions have been observed flowing into Titan's atmosphere (Hartle et al., 2006a,b) and they are thought to be deposited in the upper atmosphere around $1100 \mathrm{~km}$ (Hörst et al., 2008) where their interaction with methyl $\left(\mathrm{CH}_{3}\right)$ radicals leads to the formation of $\mathrm{CO}$. Water is instead deposited at $750 \mathrm{~km}$ due to micrometeoritic ablation (English et al., 1996) where it is photolyzed to $\mathrm{OH}$. The latter finally combines with $\mathrm{CO}$ to form $\mathrm{CO}_{2}$ and possibly other complex species.

Saturn's rings and the icy satellites that surround the giant planets, and also interplanetary dust, are probable sources of the water (oxygen) in Titan's atmosphere and recent results from INMS indicate that the plumes of Enceladus are the dominant source (e.g. Dougherty et al., 2006). Observations and models of the neutral $\mathrm{H}_{2} \mathrm{O}, \mathrm{OH}$, and $\mathrm{O}$ torus formed from the Enceladus plume show that material from Enceladus extends well beyond Titan's orbit (Melin et al., 2009; Cassidy and Johnson, 2010; Fleshman et al., 2012). Based on Herschel measurements of the Enceladus torus combined with modeling of the fate of the species within the torus, Hartogh et al. (2011) showed that the flux of $\mathrm{O} / \mathrm{O}^{+}$into Titan is consistent with an Enceladus source for the oxygen seen in Titan $\mathrm{CO}$, except for the fact that Enceladus does not seem to provide enough $\mathrm{OH} / \mathrm{H}_{2} \mathrm{O}$.

In this paper, we analyze the spectra acquired by CIRS in the far infrared spectral region in order to retrieve the water vapor vertical or spatial distribution in Titan's atmosphere. CIRS has been acquiring spectra of Titan since the beginning of the Cassini prime mission (July 2004). After 2 years of the extended mission (XM), which included the 2009 equinox, in July 2010 Cassini entered in the Solstice Mission (SM), which is scheduled to last until 2017. Since an upper limit for $\mathrm{H}_{2} \mathrm{O}$ was reported by de Kok et al. (2007a) there has been a considerable increase of the number of data collected by CIRS and significant improvements to their calibration. The increased signal to noise $(\mathrm{S} / \mathrm{N})$ ratio not only permits a definitive detection of $\mathrm{H}_{2} \mathrm{O}$ from the analysis of CIRS far infrared spectra, but it allows us to constrain its vertical and latitudinal profile.

\section{Selected dataset}

CIRS (Flasar et al., 2004) is comprised of three Focal Planes observing in the spectral range $10-1400 \mathrm{~cm}^{-1}$ with spectral resolu- tions from 0.5 to $15.5 \mathrm{~cm}^{-1}$. Focal Plane 1 detector (FP1) is characterized by a circular field of view (FOW) of $3.9 \mathrm{mrad}$. It records data in the far infrared spectral range $\left(10-600 \mathrm{~cm}^{-1}\right)$ with a spectral resolution of $0.5 \mathrm{~cm}^{-1}$, allowing us to observe the water vapor signature, and by modeling, to retrieve its abundance. Water presents its rotational lines in the CIRS FP1 spectral region up to $400 \mathrm{~cm}^{-1}$, with the strongest and most visible lines in the range positioned between 90 and $260 \mathrm{~cm}^{-1}$. We focus here on the range from 150 to $260 \mathrm{~cm}^{-1}$ for the water detection, as this is the range of maximum responsivity of FP1. At lower wavenumbers the on-board electronics of CIRS create a moving interference spike that can affect the spectrum up to $150 \mathrm{~cm}^{-1}$. Therefore, we exclude wavenumbers shorter of $150 \mathrm{~cm}^{-1}$. We use data from two different types of observations to obtain independent measurements: the far infrared on-disk integrations (FIRNADCMP) and the far infrared limb integrations (FIRLMBINT). Water is a trace species with relatively weak lines and therefore it cannot be observed in an individual spectrum. An average of a few thousand spectra of on-disk observations and a few hundred spectra of limb observations is necessary to achieve sufficient signal-to-noise (Fig. 1).

Limb observations have the FP1 Focal Plane centered around two different altitudes - hereafter limb 1 and 2 - and are therefore used to constrain the water vapor abundance in the stratosphere around 115 and $230 \mathrm{~km}$ respectively, well above the $45 \mathrm{~km}$ tropopause. Since the contribution functions of water for on-disk observations peak around $97 \mathrm{~km}$ (Fig. 2), the retrieved water vapor abundance derived from these measurements can be compared with the lowest altitude targeted by our limb integrations around $115 \mathrm{~km}$.

For the water detection and retrieval of quantitative information together with possible latitudinal variations, multiple Titan flybys must be utilized to enhance the signal. To date, 35 limb integrations of approximately $1 \mathrm{~h}$ in duration ( $\sim 60$ high-resolution spectra) have been obtained covering latitudes from $87^{\circ} \mathrm{S}$ to $80^{\circ} \mathrm{N}$. The nadir integrations are more numerous (about 92 successfully executed, of typical duration $5 \mathrm{~h}, \sim 300$ spectra) as they occur in a less contested observing time further from the Titan closest-approach period. They also have a more or less complete spatial coverage of Titan's latitudes and longitudes with an average footprint size of $\sim 15^{\circ}$ great circle arc.

We focus on one season of on-disk observations acquired from December 2004 to December 2008 (northern winter on Titan) in order to reach a compromise between obtaining a large number of spectra and a sufficiently homogeneous dataset. Inside this time period for on-disk observations acquired from a maximum distance of $300,000 \mathrm{~km}$ and with a maximum emission angle of $60^{\circ}$, we selected latitudinal bins $\left(80-45^{\circ} \mathrm{S}, 45-10^{\circ} \mathrm{S}\right.$, and $\left.0-30^{\circ} \mathrm{N}\right)$ centered around three latitudes for which observation-derived temperature profiles were available (see model description in Section 3 and Fig. 3). The number of spectra averaged in these latitudinal bins were approximately 1700, 3800 and 7000 respectively and their average emission angles were $35^{\circ}, 38^{\circ}$ and $34^{\circ}$.

CIRS limb spectra are acquired in much smaller numbers, therefore to reach a sufficient signal-to-noise ratio we consider only one average of about 320 spectra acquired from December 2004 and September 2009, encompassing the entire southern hemisphere and mid-latitudes within the range $90^{\circ} \mathrm{S}$ to $20^{\circ} \mathrm{N}$. During this time period and at these latitudes data can be considered quite homogeneous as shown in Teanby et al. (2010), therefore we model this average using a temperature profile retrieved for $15^{\circ} \mathrm{S}$. We exclude from this analysis the higher northern latitudes where the stratospheric temperature profile changes significantly. We have also selected data acquired by the spacecraft at a range less than $45,000 \mathrm{~km}$ in order to limit the size of the projected detector footprint on the limb to less than $150 \mathrm{~km}$. 

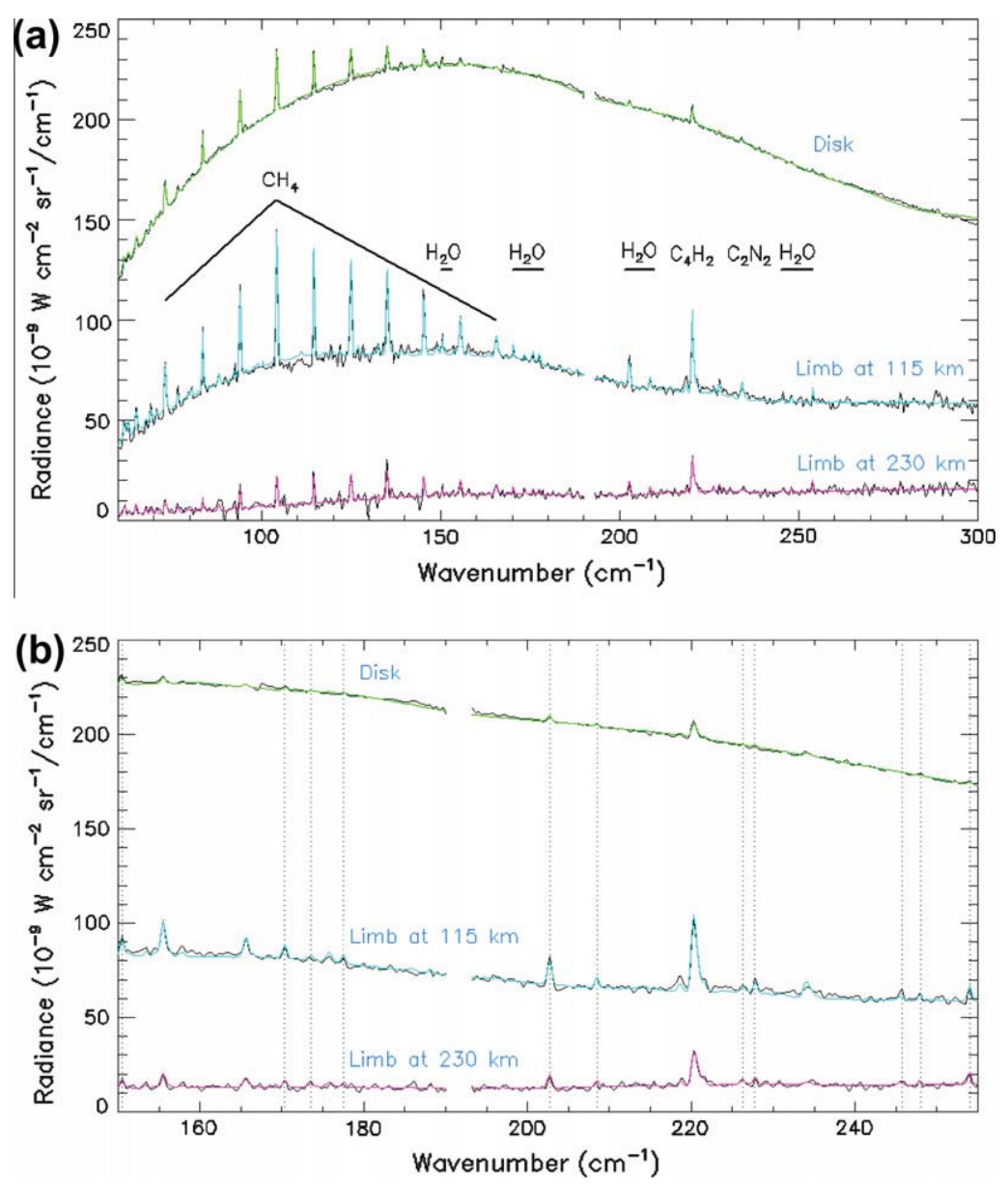

Fig. 1. (a) In black, the average of CIRS far-IR on-disk observations is plotted ( 7000 spectra acquired from December 2004 to December 2008 in the latitudinal range of 0 -

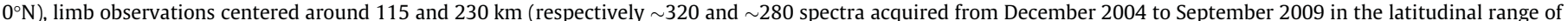

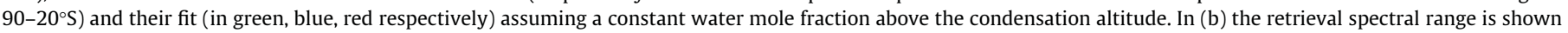

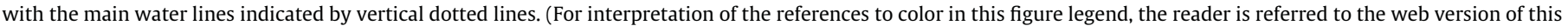
article.)

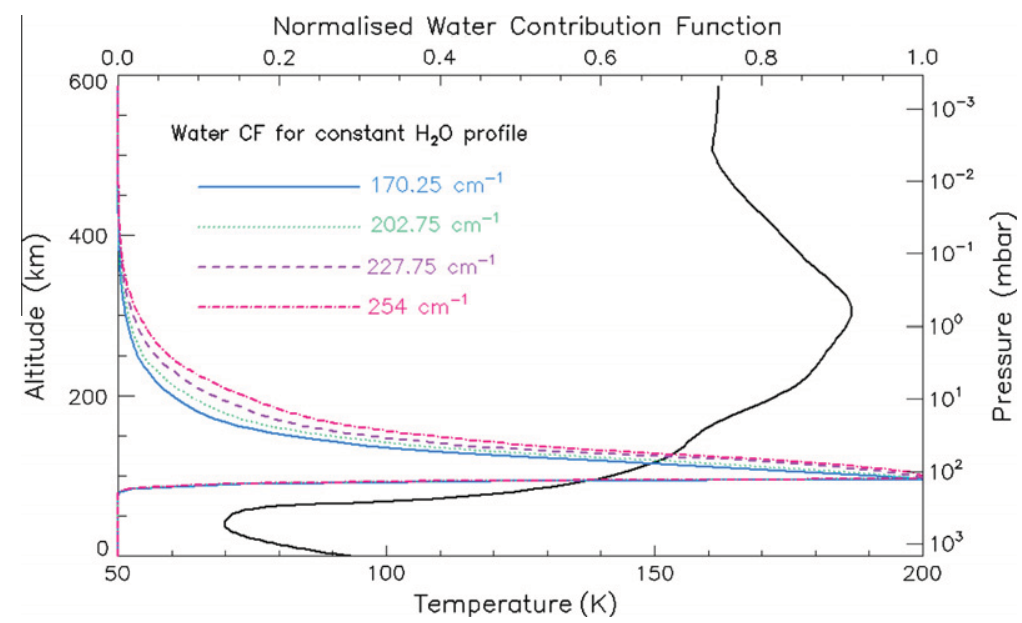

Fig. 2. Contribution functions of the different atmospheric layers to the water vapor line emission computed for four wave numbers. The solid line shows a temperature profile for $15^{\circ} \mathrm{N}$

\section{Data analysis and model}

In the selected part of the FP1 spectral range, Titan's spectrum is formed by (i) the contribution of thermal emission of the surface and atmospheric layers, (ii) the seven pairs (Anderson and Samuelson, 2011) of collision induced absorption (CIA) opacities between the main atmospheric molecules $-\mathrm{N}_{2}, \mathrm{CH}_{4}$ and $\mathrm{H}_{2}$ - due to Titan's dense lower atmosphere, (iii) the photochemical aerosol plus 


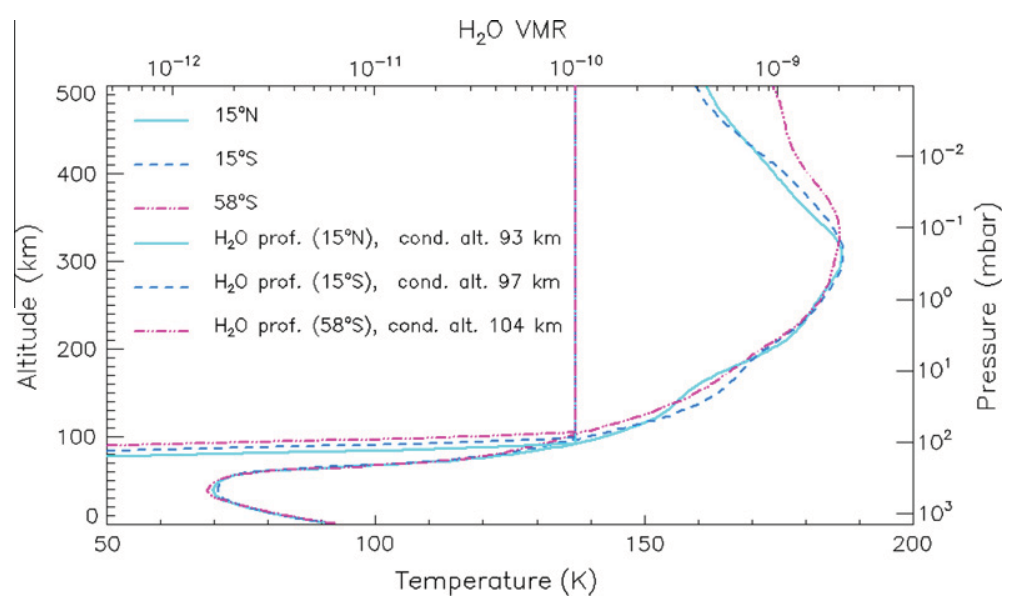

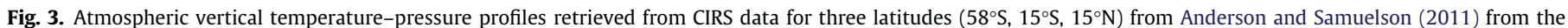

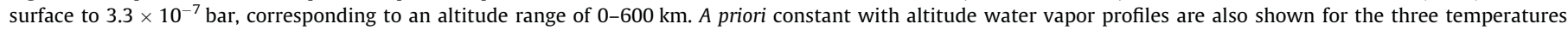

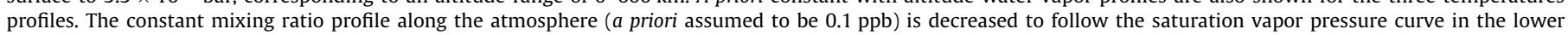

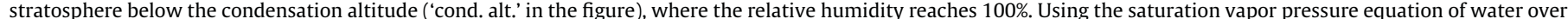

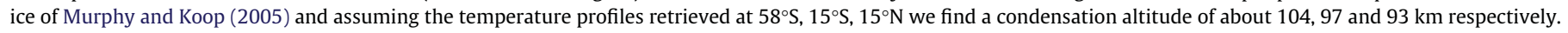

stratospheric condensates, and (iv) the ro-vibrational emission lines of atmospheric species seen by CIRS at the latitudes considered in our study: $\mathrm{CH}_{4}, \mathrm{CO}, \mathrm{H}_{2} \mathrm{O}, \mathrm{C}_{4} \mathrm{H}_{2}$.

These quantities were used as input to the NEMESIS retrieval code (Irwin et al., 2008) to perform a combination of forward model computation and retrieval scheme based on the method of optimal estimation (Rodgers, 2000). The computation of the forward model spectrum used the correlated-k approach of Lacis and Oinas (1991) and included a Hamming apodization of Full Width at Half Maximum (FWHM) of $0.5 \mathrm{~cm}^{-1}$ to reproduce the instrumental line shape. The retrieval scheme was used to optimize the fits and determine the model free parameters including the water vapor abundance. This method was successfully applied to model the FP1 spectrum in Cottini et al. (2012) to retrieve surface temperature. We solve the radiative transfer equation for 147 spherical atmospheric layers, using as a source function the thermal emission of the surface, for which a unit surface emissivity is assumed, and that of the atmospheric layers. The retrieval algorithm then iteratively computes a synthetic spectrum, compares it to the data and after applying a cost function, determines the best estimate for the physical parameters in the model - the stratospheric aerosol profile and any necessary adjustments to the temperature profile and the mole fraction of included atmospheric gases. The cost function includes two components: one that measures the quality of the fit to the spectra (similar to a $\chi^{2}$ test) and another that measures the deviation of the retrieved parameters from a set of $a$ priori quantities.

The continuum due to the CIA was calculated according to Borysow and Frommhold (1986a,b,c, 1987), Borysow (1991), and Borysow and Tang (1993). For the $\mathrm{N}_{2}-\mathrm{CH}_{4}$ pair, we used CIA coefficient values increased by $50 \%$ as required to fit the continuum of the Cassini Descent Imager Spectral Radiometer (DISR) data (Tomasko et al., 2008) and the CIRS spectra (de Kok et al., 2010).

We have modeled the haze emission/absorption using the extinction cross sections of the hazes included in de Kok et al. (2007b). Since scattering is negligible at these wavelengths for particles smaller than few microns, we have omitted it from our computations.

We have adopted the atmospheric vertical temperature-pressure profiles retrieved from CIRS data for three latitudes $\left(58^{\circ} \mathrm{S}\right.$, $15^{\circ} \mathrm{S}, 15^{\circ} \mathrm{N}$ ) from Anderson and Samuelson (2011) from the surface to $3.3 \times 10^{-7}$ bar, corresponding to an altitude range of $0-600 \mathrm{~km}$
(Fig. 3). Spectroscopic information for the gas rotational lines in the far-infrared range was extracted from the HITRAN 2004 database (Rothman et al., 2005). For $\mathrm{CH}_{4}$ we have adopted the revised mole fraction of $1.48 \%$ in the stratosphere (Niemann et al., 2010) acquired by the Gas Chromatograph Mass Spectrometer (GCMS) on the Huygens probe during in its descent to Titan's surface. In the stratosphere for $\mathrm{H}_{2}$ we assumed a uniform volume mixing ratio of $0.1 \%$ (Courtin et al., 2008). The geometry of the observations was also included in the computations.

An accurate model of the instrumental FOV is required to successfully reproduce CIRS spectra and measure water abundance. The FP1 FOV is circular and has a sensitivity with a quasi-exponential decrease from the center to the edge and a FWHM of 2.4 mrad. For on-disk spectra the homogeneity of the field of view usually permits simple modeling with a single ray calculated for the detector center. For limb spectra, we have to take into account the rapid decrease in atmospheric density with height and the variations of temperature and gas volume mixing ratio profile with altitude. In such cases, the FOV is not assumed to be uniform and a multiple ray model is required to fit the data. We modeled the FOV using the minimum number of rays for which the synthetic spectrum and the water retrieval computation results became stable; this corresponds to nine rays with a step in altitude of $25 \mathrm{~km}$. We also recomputed some of the results using 39 rays (step of $5 \mathrm{~km}$ ) in order to show a smoother limb contribution function. The spectral radiance measured by the FP1 detector is modeled by a convolution of the emerging radiance at each point in the FOV (as described in Nixon et al. (2009) and in Teanby and Irwin (2007)), weighted by a response function for CIRS FP1 detector. This beam profile was determined for CIRS FP1 (Flasar et al., 2004) using Jupiter as a point source. As $95 \%$ of the integrated response is contained in a radius of 1.95 mrad from the FOV center, the detector observes a maximum altitude range of about $70 \mathrm{~km}$.

Independent line-by-line calculations to simulate the same ondisk FP1 selections were also made using the Atmospheric Radiative Transfer (ART) code that has recently been applied to CIRS data in Coustenis et al. $(2010,2007)$. The code uses the most recent aerosol extinction dependence inferred from Vinatier et al. (2012) and temperature profiles derived by fitting the $v_{4}$ methane band at $1304 \mathrm{~cm}^{-1}$ in FP4 averages taken at similar conditions as the FP1 spectra. The spectroscopic parameters for all the observed molecules and isotopes are from GEISA 2009 (Jacquinet-Husson et al., 
2011) and HITRAN 2008 (Rothman et al., 2009). The results from these two different codes are very similar and their difference is smaller than the error bars on the data.

In Fig. 1a the on-disk and two limb observation averages are shown together with their fits. Fig. 1b shows only the spectral range used for the water line analysis.

\section{Results}

We have retrieved the water vapor abundance from both ondisk and limb data assuming different vertical profiles. These were a constant water mixing ratio profile and three vertically increasing profiles from recent photochemical models: (a) Hörst et al. (2008), (b) Wilson and Atreya (2004) and (c) Lara et al. (1996). The latter profile was adopted in Coustenis et al. (1998) for the first water detection on Titan by ISO. The constant mixing ratio profile (a priori assumed to be $0.1 \mathrm{ppb}$ ) was decreased to follow the saturation vapor pressure curve in the lower stratosphere below the altitude where the relative humidity reaches $100 \%$ (see Fig. 3). Using the saturation vapor pressure equation of water over ice of Murphy and Koop (2005) and assuming the temperature profile retrieved at $15^{\circ} \mathrm{N}$ we find a condensation altitude of $93 \mathrm{~km}$. For temperature profiles retrieved at $15^{\circ} \mathrm{S}$ and $58^{\circ} \mathrm{S}$ we find respectively condensation altitudes of $97 \mathrm{~km}$ and $104 \mathrm{~km}$. We have also computed the contribution functions - normalized inversion kernels - showing the sensitivity of each atmospheric layer to a variation of the $\mathrm{H}_{2} \mathrm{O}$ mixing ratio. These contribution functions were computed for each profile and for all of the most intense water lines in order to provide an altitude range of validity of the retrieved values (Figs. 2 and 4). Fig. 2 shows the contribution functions for ondisk observations computed at four different wave numbers; at $254 \mathrm{~cm}^{-1}$ (one of the two lines used for the ISO water retrieval) the upper shoulder of the contribution function is wider and sensitive to higher altitudes compared to the other wave numbers used for the water retrieval in this work. In our case the fit of the $254 \mathrm{~cm}^{-1}$ line improves when using a profile increasing with altitude rather than a constant profile. In Fig. 4 we show only the contribution functions computed at wavenumber $202.75 \mathrm{~cm}^{-1}$, where the most intense water line in the CIRS spectrum occurs (discounting the line at $150.5 \mathrm{~cm}^{-1}$ that is unusable due to an instrumental interference).

For water retrievals obtained using a scaled constant water profile we show the retrieved mixing ratio values at the altitude where the water contribution function peaks for the assumed profile (Table 1). For the vertical error we use the Full Width Half Maximum (FWHM) of the contribution function for the corresponding water profile. We also retrieve a scaling factor to the water profile from each of the photochemical models considered in this work (Table 1 ).

\subsection{On-disk water retrieval}

To measure the water abundance from the on-disk average (0$30^{\circ} \mathrm{N}$ ) data we first use a constant water profile. We retrieve a volume mixing ratio of $0.14 \pm 0.05 \mathrm{ppb}$ at $97 \mathrm{~km}$ (FWHM $93-130 \mathrm{~km}$ ). This value corresponds to a surface-normalized $\mathrm{H}_{2} \mathrm{O}$ total column density of $3.7 \pm 1.3 \times 10^{14}$ molecules $/ \mathrm{cm}^{2}$.

The largest source of error for the on-disk observations is due to the fact that the maximum of the contribution function occurs in the region where the water abundance is rapidly changing due to condensation. Other sources of error include small variations of the temperature profile in the stratosphere, random noise from the detectors, and a small dependence in altitude sensitivity with wavenumber.

We also fit the water lines for the three other water vertical distribution profiles (Lara et al., 1996; Wilson and Atreya, 2004; Hörst et al., 2008) and obtained the necessary scale factors to fit the data, which are shown in Table 1 . These values, ranging between 0.11 and 0.63 times the considered profiles, show the retrieved water mole fraction to be less than predicted from these previous models.

We have analyzed two additional latitudinal bins from $45^{\circ} \mathrm{S}$ to $10^{\circ} \mathrm{S}$ and from $80^{\circ} \mathrm{S}$ to $45^{\circ} \mathrm{S}$, centered on the latitudes corresponding to the temperature profiles previously retrieved from CIRS at $15^{\circ} \mathrm{S}$ and $58^{\circ} \mathrm{S}$ respectively (Anderson and Samuelson, 2011). See Fig. 3. The observed water mixing ratio indicates the absence of any significant latitudinal variations within the error bars in these
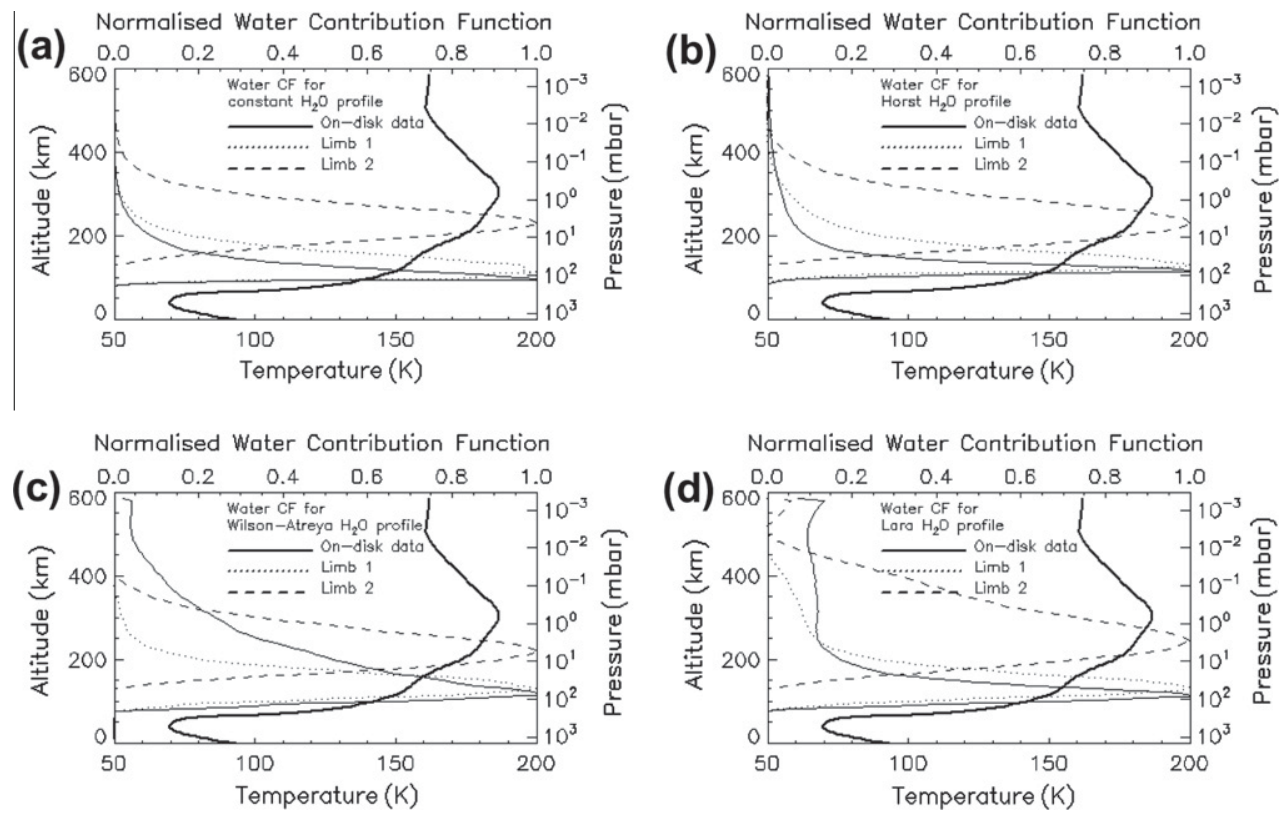

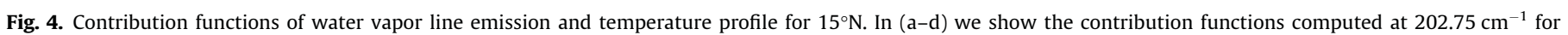

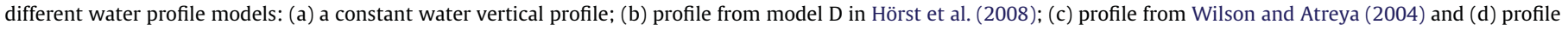
from Lara et al. (1996). 
Table 1

Water vapor abundance results.

\begin{tabular}{|c|c|c|c|}
\hline Water vapor retrieved mole fractions & On-disk average $\left(0-30^{\circ}\right) \mathrm{N}$ & Limb retrieval 1 & Limb retrieval 2 \\
\hline Constant VMR profile & $(0.14 \pm 0.05) \mathrm{ppb}$ at $97_{-4}^{+33} \mathrm{~km}$ & $(0.13 \pm 0.04) \mathrm{ppb}$ at $115_{-20}^{+50} \mathrm{~km}$ & $(0.45 \pm 0.15) \mathrm{ppb}$ at $230_{-40}^{+45} \mathrm{~km}$ \\
\hline Scaling factor to $\mathrm{H}_{2} \mathrm{O}$ profile from Hörst (model D) & $0.18 \pm 0.05$ at $118_{-12}^{+20} \mathrm{~km}$ & $0.14 \pm 0.05$ at $129_{-17}^{+45} \mathrm{~km}$ & $0.23 \pm 0.07$ at $232_{-60}^{+67} \mathrm{~km}$ \\
\hline Scaling factor to $\mathrm{H}_{2} \mathrm{O}$ profile from Wilson-Atreya & $0.14 \pm 0.05$ at $118_{-22}^{+85} \mathrm{~km}$ & $0.13 \pm 0.05$ at $129_{-24}^{+46} \mathrm{~km}$ & $0.18 \pm 0.08$ at $222_{-53}^{+62} \mathrm{~km}$ \\
\hline Scaling factor to $\mathrm{H}_{2} \mathrm{O}$ profile from Lara & $0.48 \pm 0.07$ at $115_{-30}^{+20} \mathrm{~km}$ & $0.63 \pm 0.07$ at $133_{-27}^{+42} \mathrm{~km}$ & $0.45 \pm 0.08$ at $247_{-100}^{+33} \mathrm{~km}$ \\
\hline
\end{tabular}

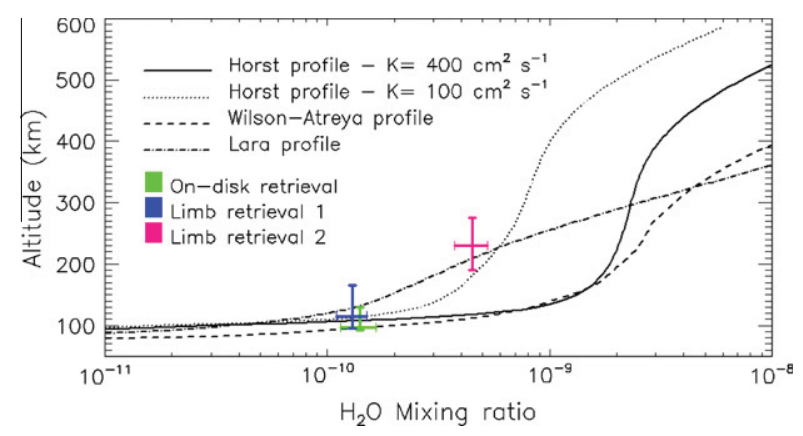

Fig. 5. Water vapor mole fraction retrieved from CIRS on-disk and two limb observations assuming a water profile constant with altitude over the condensation level. Water profiles from three photochemistry models are also shown for comparison. The Hörst et al. (2008) water vapor profile was derived assuming two different eddy diffusion coefficients $-100 \mathrm{~cm}^{2} \mathrm{~s}^{-1}$ and $400 \mathrm{~cm}^{2} \mathrm{~s}^{-1}$ (the second being the one recommended in their model; dotted curve plus solid curve). Also the profiles in Wilson and Atreya (2004; dashed curve) and in Lara et al. (1996; dotdash curve).

latitude ranges. It should be stressed that in this work we did not analyze the water stratospheric content at high northern latitudes that were experiencing winter during this time period. To model spectra at these latitudes for a large average is particularly complex since the stratospheric temperature was changing quickly with latitude. In addition the temperature profiles for high northern latitudes are not yet available for the lower stratospheric region sensed by the CIRS water lines.

The results obtained simultaneously for on-disk data using the independent line-by-line ART code simulations of the same FP1 selections confirm the retrieved water vapor value reported above within the error bars.

\subsection{Limb water retrieval}

The measurement of water vapor obtained by modeling the limb 1 spectrum under the assumption of a constant mixing ratio profile is equal to $0.13 \pm 0.04 \mathrm{ppb}$. According to the position of the peak of the corresponding contribution function, the radiance mostly originates from a region centered at $115 \mathrm{~km}$ (FWHM 95$165 \mathrm{~km}$ ). Modeling the limb 2 spectrum we retrieved a water mixing ratio using a constant water profile of $0.45 \pm 0.15 \mathrm{ppb}$ at an altitude of $230 \mathrm{~km}$ (FWHM 190-275 km). These values indicate an increase of the water mole fraction with altitude in the stratosphere from $115 \mathrm{~km}$ to $230 \mathrm{~km}$ of about three times.

The scaling factors to the model water profiles obtained from the two types of limb retrievals are shown in Table 1 and again illustrate the smaller amount of stratospheric water vapor detected by CIRS with respect to the ones predicted by the models considered in this study.

\section{Conclusions}

In this work we modeled CIRS data using a constant-withheight water vapor profile and assigned the retrieved mixing ratio to the altitude where the contribution function peaks.
By combining on-disk and limb observations we are able to constrain the vertical profile of water in the stratosphere from $12 \mathrm{mbar}$ to $10^{-3}$ mbar, corresponding to altitudes between 93 and $280 \mathrm{~km}$ (considering the widths of the contribution functions).

In Fig. 5 we compare our water vapor retrieved values with the models of (Lara et al., 1996; Wilson and Atreya, 2004; Hörst et al., 2008).

The measurement of the stratospheric vertical profile of water adds useful constraints to the photochemical models of Titan's atmosphere. Qualitatively, the increase of the water mixing ratio with altitude is in agreement with an external source of oxygen and a lower altitude sink due to condensation. However, quantitatively, our retrieved abundance seems to be less (from $\sim 0.1$ to 0.6) than predicted from the models considered in this work (see Table 1). We also observe that since the scaling factors to the photochemical models (a)-(c) in Table 1 are slightly different for the two limb altitudes, it implies that these models might have a slope for $\mathrm{H}_{2} \mathrm{O}$ not quite consistent with CIRS data. However, due to the rapid variation of the water vapor mole fraction with altitude in the atmospheric region where water freezes, and where CIRS is actually observing (on-disk and limb 1 spectra), we should be cautious in assigning a slope to the water profile.

Coustenis et al. (1998) fitted the ISO data with a scaling factor to the Lara et al. (1996) water profile of $0.4_{-0.2}^{+0.3}$. We fitted the CIRS data assuming the same profile multiplied by a scaling factor of $0.48 \pm 0.07$ (corresponding to a water column density of $3.8 \pm 1.0 \times 10^{14}$ molecules $\mathrm{cm}^{-2}$ ). This result agrees with the scaling factor from the ISO analysis although this agreement may be fortuitous because most of the ISO emission originates from above $300 \mathrm{~km}$, with the contribution functions peaking around $400 \mathrm{~km}$ (Fig. 3 of Coustenis et al. (1998)). Since the beam size of ISO was much larger than Titan there is a strong emission from the limb occurring at high altitudes where 0.4 times the Lara et al. (1996) profile is used. Due to its higher spatial resolution, the contribution functions for the CIRS nadir selection using the Lara et al. (1996) profile cover the range $95-145 \mathrm{~km}$ (at half maximum).

ISO also retrieved a water vapor abundance of $0.4 \mathrm{ppb}$ assuming a constant mole fraction above the condensation level (Coustenis et al., 1998); we assign the same relative error bars as those derived by ISO for the scaled Lara et al. (1996) profile: +0.3 and -0.2 . From CIRS on-disk observations we retrieved a volume mixing ratio of $0.14 \pm 0.05 \mathrm{ppb}$ around $97 \mathrm{~km}$ for latitudes $0-30^{\circ} \mathrm{N}$. Our retrieval is only marginally consistent with the ISO determination of $0.4_{-0.2}^{+0.3} \mathrm{ppb}$ above the water vapor condensation altitude. However it is necessary to make the assumption that the ISO results pertain to the same condensation altitude that we retrieve because unfortunately it is not reported in the ISO paper.

We now discuss how our retrieved water abundances compare with current models of oxygen photochemistry on Titan. The retrieved scaling factors are all less than one, implying that there are still uncertainties in our understanding of oxygen processes on Titan.

In photochemical models of Titan prior to 2000 (e.g. Lara et al., 1996), in order to allow atmospheric production of CO, it was postulated that $\mathrm{CO}$ could be produced through a chemical reaction between $\mathrm{OH}$ (available from $\mathrm{H}_{2} \mathrm{O}$ influx into the upper atmosphere) 
and $\mathrm{CH}_{3}$. However, it was emphasized by Wong et al. (2002) that this reaction does not produce $\mathrm{CO}$ as previously assumed but instead it produces $\mathrm{H}_{2} \mathrm{O}$ (Pereira et al., 1997).

Hence an influx of $\mathrm{H}_{2} \mathrm{O}$ or $\mathrm{OH}$ does not produce any significant abundance of $\mathrm{CO}$ and therefore $\mathrm{CO}_{2}$ can be produced by an $\mathrm{H}_{2} \mathrm{O}$ influx only with $\mathrm{CO}$ already present $\left(\mathrm{OH}+\mathrm{CO} \rightarrow \mathrm{CO}_{2}+\mathrm{H}\right)$. For this reason these models were unable to reproduce the observed $\mathrm{CO}$ abundance and were substituted by other models that suggest the existence of primordial $\mathrm{CO}$ in the atmosphere (Wilson and Atreya, 2004), or instead CO produced in the atmosphere using an external influx of $\mathrm{O}^{+}$rather than $\mathrm{H}_{2} \mathrm{O}$ or $\mathrm{OH}$ (Hörst et al., 2008).

In the pre-Cassini model of Wilson and Atreya (2004) water is photolyzed to $\mathrm{OH}$, which combines with $\mathrm{CO}$ to form $\mathrm{CO}_{2}$ and other complex species. In this model, $\mathrm{CO}$ is assumed to be primordial on Titan and the water abundance profile derives from the amount necessary to form the observed $\mathrm{CO}_{2}$. This assumption was challenged by the Hörst et al. (2008) model, in which oxygen species are assumed to arrive from outside the Moon and form carbon monoxide as well as carbon dioxide in the atmosphere. The values of the input fluxes of $\mathrm{O}$ and $\mathrm{OH}$ were adjusted to reproduce the observed abundances of $\mathrm{CO}$ and $\mathrm{CO}_{2}$. In the Hörst et al. (2008) model water profiles were produced for six different values (from $K_{0}=100 \mathrm{~cm}^{2} \mathrm{~s}^{-1}$ to $K_{0}=1000 \mathrm{~cm}^{2} \mathrm{~s}^{-1}$ ) of the eddy coefficient in the lower atmosphere, since the stratospheric abundances of photochemically produced species are highly dependent on this parameter. As shown in Fig. 5, the water abundance retrieved in our study is best fit by the water profile with the lowest eddy diffusion coefficient value considered in their model $\left(K_{0}=100 \mathrm{~cm}^{2} \mathrm{~s}^{-1}\right)$. This is lower than the value $\left(K_{0}=400 \mathrm{~cm}^{2} \mathrm{~s}^{-1}\right)$ they identified as best reproducing CIRS observations of hydrocarbon species and adopted in this work for comparison with our retrievals. However our results show that even the Hörst model with $K_{0}=100 \mathrm{~cm}^{2} \mathrm{~s}^{-1}$ still has excessive water at the altitudes of our measurements.

Therefore, our work clearly points towards further refinement of oxygen chemistry in photochemical models of Titan's atmosphere.

\section{Acknowledgments}

Valeria Cottini is supported by the NASA Postdoctoral Program. Thanks to S. Hörst, E. Wilson and S. Atreya for providing their photochemical water profiles for comparison, and to Paul Romani for water chemistry discussions. The US-based authors were funded by the NASA Cassini Mission during the period in which this work was performed. N. Teanby was supported by the Leverhulme Trust and the UK Science and Technology Facilities Council.

\section{References}

Anderson, C.M., Samuelson, R.E., 2011. Titan's aerosol and stratospheric ice opacities between 18 and $500 \mu \mathrm{m}$ : Vertical and spectral characteristics from Cassini CIRS. Icarus 212, 762-778.

Bjoraker, G., Achterberg, R., Anderson, C., Samuelson, R., Carlson, R., Jennings, D., 2008. American Astronomical Society. DPS Meeting \#40, \#31.12, Bulletin of the AAS, 40, 448.

Borysow, A., 1991. Modelling of collision-induced infrared-absorption spectra of $\mathrm{H}_{2}-\mathrm{H}_{2}$ pairs in the fundamental band at temperatures from $20 \mathrm{~K}$ to $300 \mathrm{~K}$. Icarus $92(2), 273-279$.

Borysow, A., Frommhold, L., 1986a. Theoretical collision-induced rototranslational absorption spectra for modeling Titan's atmosphere $-\mathrm{H}_{2}-\mathrm{N}_{2}$ pairs. Astrophys. J. 303, 495-510.

Borysow, A., Frommhold, L., 1986b. Theoretical collision-induced rototranslational absorption spectra for the outer planets- $\mathrm{H}_{2}-\mathrm{CH}_{4}$ pairs. Astrophys. J. 304, 849865.

Borysow, A., Frommhold, L., 1986c. Collision-induced rototranslational absorption spectra of $\mathrm{N}_{2}-\mathrm{N}_{2}$ pairs for temperatures from 50 to 300 K. Astrophys. J. 311, 1043-1057.

Borysow, A., Frommhold, L., 1987. Collision-induced rototranslational absorption spectra of $\mathrm{CH}_{4}-\mathrm{CH}_{4}$ pairs at temperatures from 50 to $300 \mathrm{~K}$. Astrophys. J. 318, 940-943.

Borysow, A., Tang, C., 1993. Far infrared CIA spectra of $\mathrm{N}_{2}-\mathrm{CH}_{4}$ pairs for modelling of Titan's atmosphere. Icarus 105, 175-183.
Cassidy, T.A., Johnson, R.E., 2010. Collisional spreading of Enceladus' neutral cloud. Icarus 209 (2), 696-703.

Cottini, V. et al., 2012. Spatial and temporal variations in Titan's surf ace temperatures from Cassini CIRS observations. Planet. Space Sci. 60, 62-71.

Courtin, R.D., Sim, C., Kim, S., Gautier, D., Jennings, D.E., 2008. Latitudinal variations of tropospheric $\mathrm{H}_{2}$ on Titan from the Cassini CIRS investigation. Bull. Am. Astron. Soc. 40, 446.

Coustenis, A. et al., 1998. Evidence for water vapor in Titan's atmosphere from ISO/ SWS data. Astron. Astrophys. 336, L85-L89.

Coustenis, A. et al., 2007. The composition of Titan's stratosphere from Cassini/CIRS mid-infrared spectra. Icarus 189, 35-62.

Coustenis, A. et al., 2010. Titan trace gaseous composition from CIRS at the end of the Cassini-Huygens prime mission. Icarus 207, 461-476.

Cui, J., Yelle, R.V., Vuitton, V., Waite, J.H., Kasprzak, W.T., Niemann, H.B., Gell, D., Borggren, N., Magee, B., Müller-Wodarg, I.C.F, 2009. Analysis of Titan's neutral upper atmosphere from Cassini ion neutral mass spectrometer measurements. Icarus 200, 581-615.

de Kok, R. et al., 2007a. Oxygen compounds in Titan's stratosphere as observed by Cassini CIRS. Icarus 186, 354-363.

de Kok, R., Irwin, P.G.J., Teanby, N.A., Nixon, C.A., Jennings, D.E., Fletcher, L., Howett C., Calcutt, S.B., Bowles, N.E., Flasar, F.M., Taylor, F.W., 2007b. Characteristics of Titan's stratospheric aerosols and condensate clouds from Cassini CIRS farinfrared spectra. Icarus 191 (1), 223-235.

de Kok, R., Irwin, P.G.J., Teanby, N.A., 2010. Far-infrared opacity sources in Titan's troposphere reconsidered. Icarus 209 (2), 854-857.

Dougherty, M.K. et al., 2006. Identification of a Dynamic Atmosphere at Enceladus with the Cassini Magnetometer. Science 311, 1406-1409.

English, M.A., Lara, L.M., Lorenz, R.D., Ratcliff, P.R., Rodrigo, R., 1996. Ablation and chemistry of meteoric materials in the atmosphere of Titan. Adv. Space Res. 17, $157-160$.

Flasar, F.M. et al., 2004. Exploring the Saturn system in the thermal infrared: The Composite Infrared Spectrometer. Space Sci. Rev. 115, 169-297.

Fleshman, B.L., Delamere, P.A., Bagenal, F., Cassidy, T., 2012. The roles of charge exchange and dissociation in spreading Saturn's neutral clouds. J. Geophys. Res., in press. http://dx.doi.org/10.1029/2011JE003996.

Gurwell, Mark A., 2004. Submillimeter Observations of Titan: Global Measures of Stratospheric Temperature, $\mathrm{CO}, \mathrm{HCN}, \mathrm{HC}_{3} \mathrm{~N}$, and the Isotopic Ratios ${ }^{12} \mathrm{C} /{ }^{13} \mathrm{C}$ and ${ }^{14} \mathrm{~N} /{ }^{15} \mathrm{~N}$. Astrophys. J. 616, L7-L10.

Hartle, R.E. et al., 2006a. Preliminary interpretation of Titan plasma interaction as observed by the Cassini Plasma Spectrometer: Comparisons with Voyager 1. Geophys. Res. Lett., 33.

Hartle, R.E. et al., 2006b. Initial interpretation of Titan plasma interaction as observed by the Cassini Plasma Spectrometer: Comparisons with Voyager 1. Planet. Space Sci. 54, 1211-1224.

Hartogh, P. et al., 2011. Direct detection of the Enceladus water torus with Herschel. Astron. Astrophys. 532 (Article ID L2).

Hidayat, T. et al., 1998. Millimeter and submillimeter heterodyne observations of Titan: The vertical profile of carbon monoxide in its stratosphere. Icarus 133 , 109-133.

Hörst, S.M., Vuitton, V., Yelle, R.V., 2008. Origin of oxygen species in Titan's atmosphere. J. Geophys. Res. 113, E10.

Irwin, P.G.J. et al., 2008. The NEMESIS planetary atmosphere radiative transfer and retrieval tool. J. Quant. Spectrosc. Radiat. Trans. 109, 1136-1150.

Jacquinet-Husson, N. et al., 2011. The 2009 edition of the GEISA spectroscopic database. J. Quant. Spectrosc. Radiat. Trans. 112, 2395-2445.

Lacis, A.A., Oinas, V., 1991. A description of the correlated k distributed method for modeling nongray gaseous absorption, thermal emission, and multiple scattering in vertically inhomogeneous atmospheres. J. Geophys. Res. 96, 9027-9063.

Lara, L.M., Lellouch, F., Lopez-Moreno, J.J., Rodrigo, R., 1996. Vertical distribution of Titan's atmospheric neutral constituents. J. Geophys. Res. 101 (23), 261.

Lutz, B.L., de Bergh, C., Owen, T., 1983. Titan - Discovery of carbon monoxide in its atmosphere. Science 220, 1374-1375.

Melin, H., Shemansky, D.E., Liu, X., 2009. The distribution of atomic hydrogen and oxygen in the magnetosphere of Saturn. Planet. Space Sci. 57 (14-15), 17431753.

Murphy, D.M., Koop, T., 2005. Review of the vapour pressures of ice and supercooled water for atmospheric applications. Q. J. R. Meteorol. Soc. 131, 1539-1565.

Niemann, H.B. et al., 2010. The composition of Titan's lower atmosphere and simple surface volatiles as measured by the Cassini-Huygens probe gas chromatograph mass spectrometer experiment. J. Geophys. Res. 115, E12006.

Nixon, C.A. et al., 2009. Infrared limb sounding of Titan with the Cassini Composite Infrared Spectrometer: Effects of the mid-IR detector spatial responses. Appl. Opt. 48, 1912-1925.

Pereira, R.A., Baulch, D.L., Pilling, M.J., Robertson, S.H., Zeng, G., 1997. Temperature and pressure dependence of the multichannel rate coefficients for the $\mathrm{CH}_{3}+\mathrm{OH}$ system. J. Phys. Chem. A 101, 9681-9693.

Rodgers, C.D., 2000. Inverse Methods for Atmospheric Sounding: Theory and Practice. World Scientific, Singapore.

Rothman, L.S. et al., 2005. The HITRAN 2004 molecular spectroscopic database. J. Quant. Spectrosc. Radiat. Trans. 96, 139-204.

Rothman, L.S. et al., 2009. The HITRAN 2008 molecular spectroscopic database. J Quant. Spectrosc. Radiat. Trans. 110, 533-572.

Samuelson, R.E. et al., 1983. $\mathrm{CO}_{2}$ on Titan. J. Geophys. Res. 88, 8709-8715.

Teanby, N.A., Irwin, P.G.J., 2007. Quantifying the effect of finite field-of-view size on radiative transfer calculations of Titan's limb spectra measured by Cassini-CIRS Astrophys. Space Sci. 310, 293-305. 
Teanby, N.A. et al., 2009. Titan's stratospheric $\mathrm{C}_{2} \mathrm{~N}_{2}, \mathrm{C}_{3} \mathrm{H}_{4}$, and $\mathrm{C}_{4} \mathrm{H}_{2}$ abundances from Cassini/CIRS far-infrared spectra. Icarus 202, 620-631.

Teanby, N.A., Irwin, P.G.J., de Kok, R., Nixon, C.A., 2010. Seasonal changes in Titan's polar trace gas abundance observed by cassini. The Astrophysical Journal Letters 724 (2010), L84.

Tomasko, M.G. et al., 2008. A model of Titan's aerosols based on measurements made inside the atmosphere. Planet. Space Sci. 56, 669-707.
Vinatier, S., Rannou, P., Anderson, C.M., Bézard, B., de Kok, R., Samuelson, R.E., 2012. Optical constants of Titan's stratospheric aerosols in the $70-1500 \mathrm{~cm}^{-1}$ spectral range constrained by Cassini/CIRS observations. Icarus 219, 5-12.

Wilson, E.H., Atreya, S.K., 2004. Current state of modeling the photochemistry of Titan's mutually dependent atmosphere and ionosphere. J. Geophys. Res. 109, E6.

Wong, A., Morgan, C.G., Yung, Y.L., Owen, T., 2002. Evolution of CO on Titan. Icarus 155 (2), 382-392. 